\title{
Design and Research of Control System of the Large-Scale Accumulator Blow Molding Machine Based on PLC
}

\author{
Wei-min $\mathrm{LI}^{1, \text { a }}$, Yang XU ${ }^{1, \mathrm{~b}}$ Dian-yi FENG ${ }^{1, \mathrm{c}}$ Meng-de ZHOU ${ }^{1, \mathrm{~d}}$ \\ ${ }^{1}$ Faculty of Mechanical Engineering and Automation, Liaoning University of Technology, Jinzhou \\ 121001, China \\ aLiwmqh@163.com, ${ }^{b}$ Xuyangon@163.com ${ }^{\mathrm{d}}$,Mengde.521@163.com.
}

Keywords: Large-scale blow molding machine, Wall thickness control system, HMI, PLC, Cubic spline interpolation

\begin{abstract}
On the basis of process flow of the large-scale blow molding machine, make PLC as the control center, touch screen as the human-computer interface, this passage introduce the overall plan of the control system, hardware configuration and design procedures of the software, make communication technology come true between PLC and HMI with MPI, Use PID technology, make the wall thickness come true by the way of cubic spline interpolation.
\end{abstract}

\section{Introduction}

With the energy crisis appears continuously, use of blow molding products increasingly abroad. Now, the middle and small scale of blow molding devices become more mature. But as the plastics industry explosion and need for the large-scale cavity containers, especially the large capacity products, become the emphasis of the development by enterprise domestic and overseas. As the result of productive technology of the large-scale blow molding machine is too complex, requires more distribution of I/O points and demand of the control system more complex, the way of the control system is prematuration. This passage give the example as capacity 200L, use HMI as the upper computer and PLC as the lower computer in control system, through man-machine interaction, make the field data collected, monitored and modification in real time.

\section{Technological Process of Blow Molding Machine}

Productive technology of the blow molding machine is complex, the process flow as fig 1.Put the granuliform polypropylene and polyethylene raw materials from feeding funnel, extrusion motor start, then the screw moves and materials load start. Start Electromagnetic heating, raw materials in the heated barrel are heated and extruding turned into molten state then squeezed into accumulator head at the same time. When the materials reach position that the Linear Ball Bearing Series setted, it gives out the signal, piston of the accumulator push down and the parison is extrusion. Parison get into the mould, then compound die and blowing, refrigeration and pressure maintaining, at last get out the products with the mechanical arm and get the end product through manual deburring, the wastes through the crusher's disposing are fed into the feeding funnel for the next operating cycle. Therefore this system is a sequential control system. To make the surge solenoid valves and the pneumatic solenoid valves' open and close or the motors' foreward and inversion, start and stop with PLC in order to accomplish the movement such as mold opening and closing, blowing needles' open and close, manipulator's movement etc. 


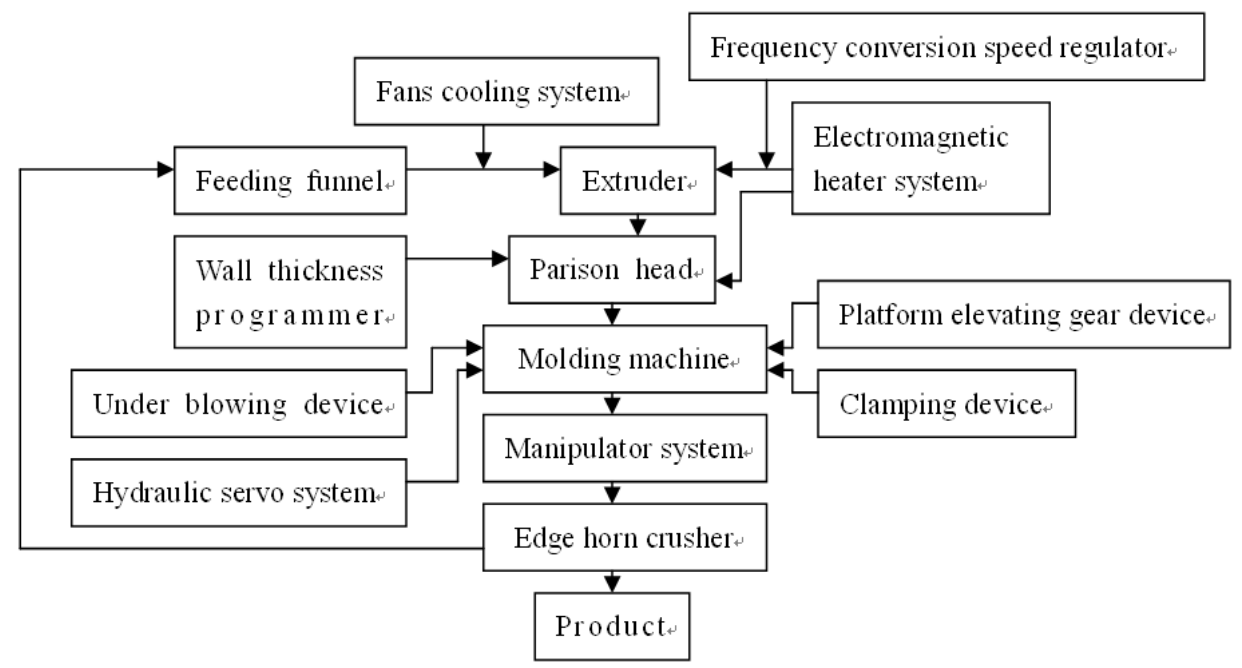

Fig.1 The process flow of extrusion blow molding

\section{Hardware Construction of Blow Molding Machine}

Hardware configuration of control system.Programmable logic controller(PLC)make the microprocessor as its core and the logic control as its foundation, it can provides logic control, timekeeping, counting, numeration, ramification and I/O control function, it has a simple structure and convenient to use. With PLC in the sequential control system of mechanisms and devices, it can enhance the system's reliability. Take into consideration of the industrial art that need to achieve the control requirement, it needs DI84 and DO38, so we choose the S7-300 series CPU313C. The hardware configuration of the control system lies in fig.2.

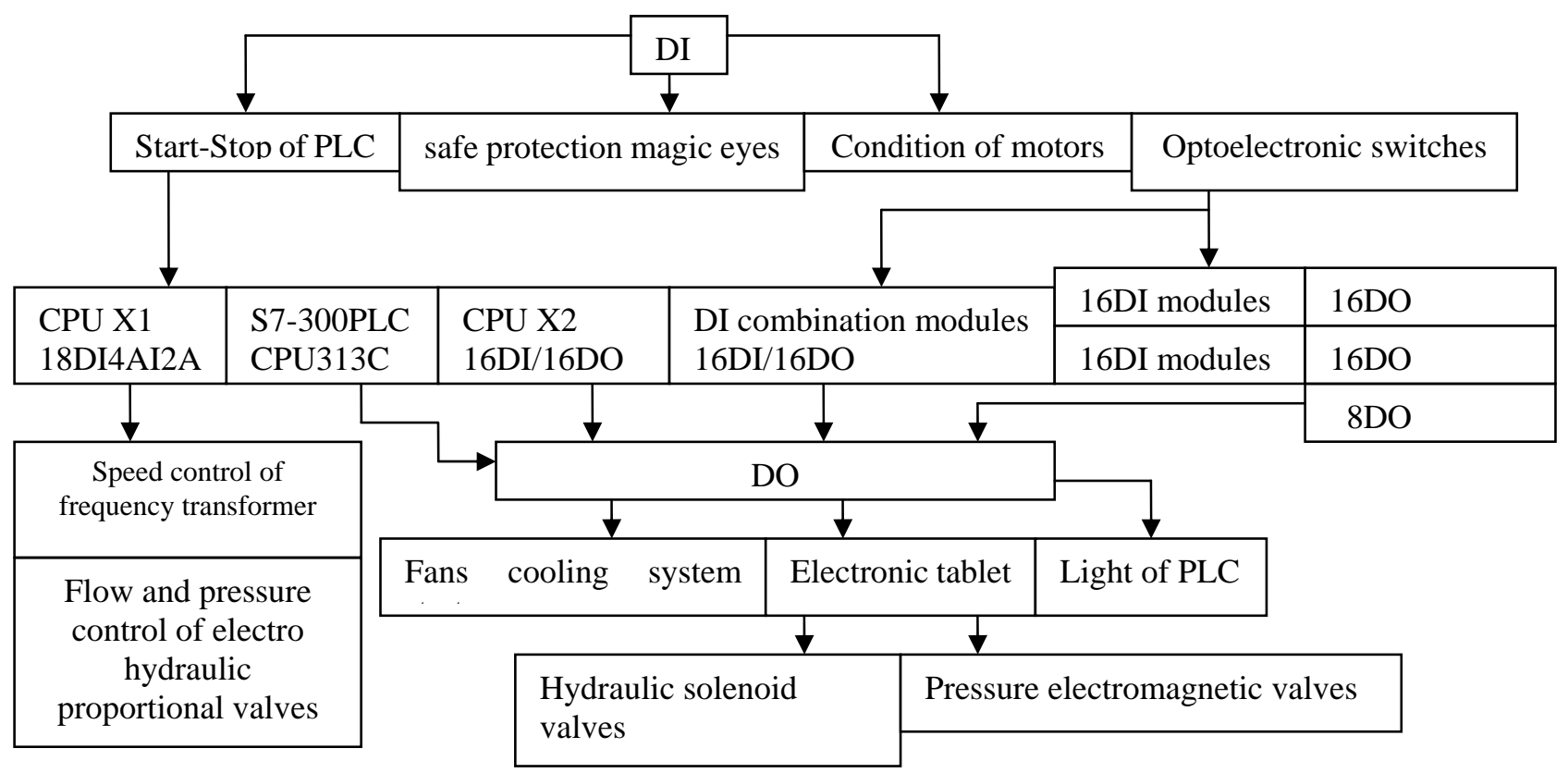

Fig.2 Hardware configuration control system of PLC

Distribution of the power supply system.As each part different demand for the power supply, the hardware system is divide into these parts below, such as cabinet lighting, PLC, touch screen, draught fan and voiced light alarm etc as fig.3. 


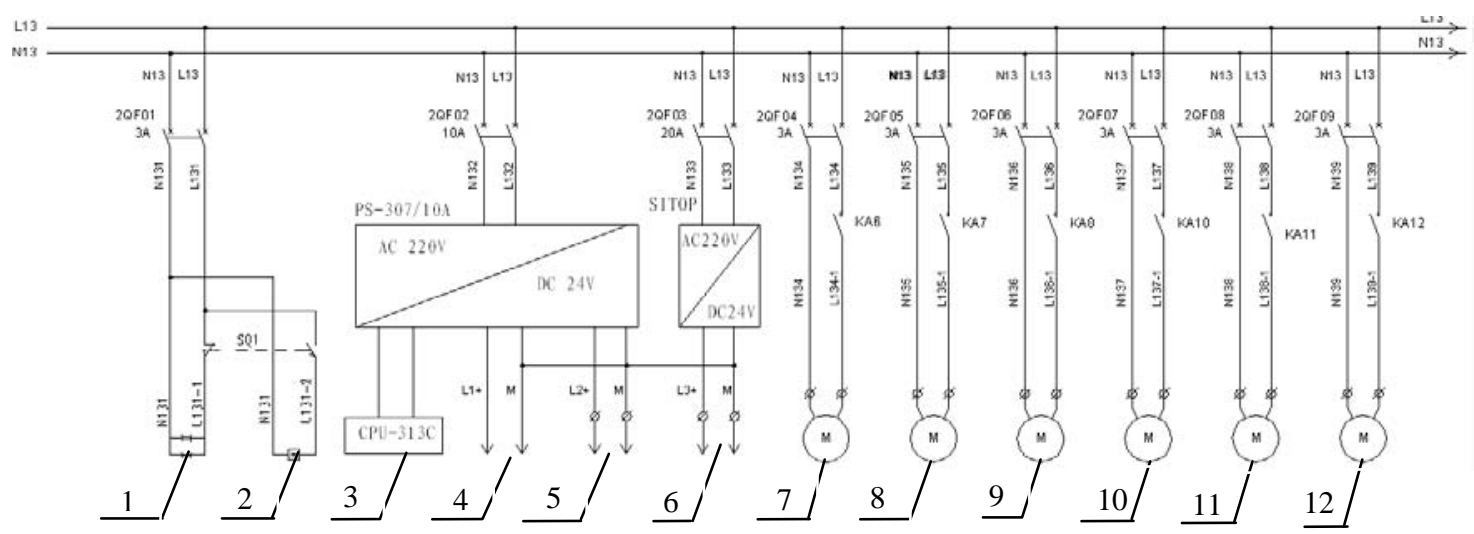

Fig.3 Distribution of the power supply system

1-Cabinet lighting, 2-Fans in cabinet, 3-PLC CPU

4-Power of sensors, 5-Power of HMI, 6-Executive component drive,7-12-Cooling fans

Characteristic of the hardware system. As the complicacy of the system's productive technology, a large amount of DI and DO, and the communication between PLC and touch screen is the core in control system.

Large amount digital quantity I/O.As complicacy of the system's productive technology, that means it needs a large amount sensors, limit switches, solenoid valves to feed back location and movement. This system needs DI84, DO38 and 4 pieces of electron plates. DO can connect with the solenoid valves through electron plates. The fig. 4 is wiring scheme of DO and electron plates.Fig.5 is wiring scheme of DI in the PLC.

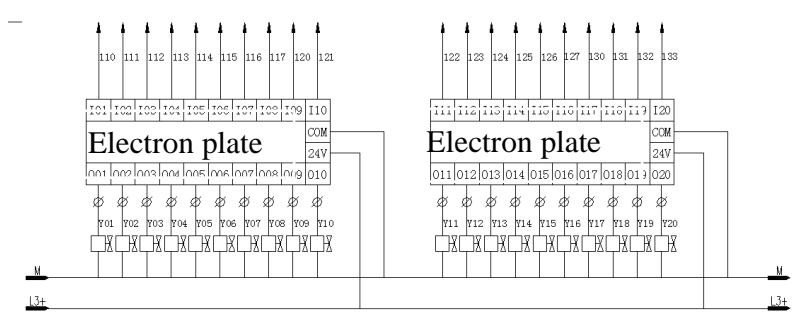

Fig. 4 Wiring scheme of the DO and electron plates

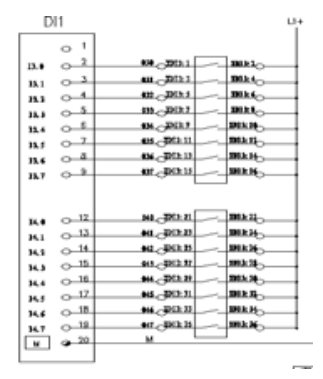

Fig. 5 wiring scheme of DI

Communication between PLC and touch screen.MPI (Multi Point Interface) is a kind of classified treaty used for communication between PLC. Its main advantage is the CPU can build communication with mulit-devices at the same time. This system the communication between PLC and HMI is depend on MPI. The other communication objects like transducer, temperature control modules can through the standard serial interface RS-485 and HMI for communication.Fig.6 is the communication network arrangement plan.

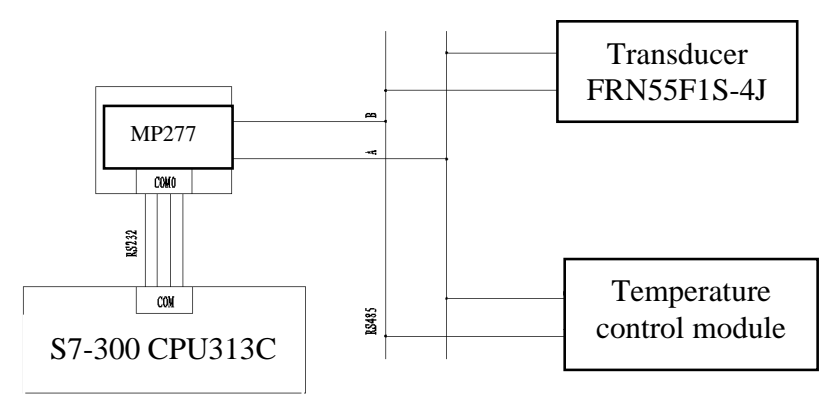

Fig. 6 Communication network arrangement plan

The parison wall thickness programmer based on PID.The blow molding products have been set the minimum wall thickness as requirement for strength. Wall thickness of parison too weak may cause the parison broken in the process of blowing or lack of products' intensity.If the wall thickness too thick may cause the products overweight and unnecessary waste raw materials so that may 
increase the cost of production. Therefore, Parison wall thickness programmer is a major measure to enhance the products quality and it is also one of key technology of products.

Wall thickness control technology falls into two forms: axial wall thickness control technique and radial wall thickness control technique. The setting points of wall thickness graph must be continuous and derivable, otherwise there will be loop fringes in axial wall thickness distribution or axial fringes in radial wall thickness distribution. This passage adopt PID closed-loop control technique combined with cubic spline interpolation to accomplish the control in parison wall thickness.

Operating principle and form of blow molding wall thickness control system. The basic operation principle of wall thickness control system is that to control the opening of die through electro-hydraulic proportional valves so that can control the change of wall thickness and make wall thickness uniformity. The wall thickness control system adopt closed loop feedback technique. Its constituent parts include wall thickness programmer, electro-hydraulic proportional valves, servo hydraulic cylinders, and linear displacement sensors. Users can set the parison wall thickness axial changing curves on the panel of wall thickness programmer. This programmer export the voltage and electric current signal to electro-hydraulic proportional valves based on the curve and electro-hydraulic proportional valves drive servo hydraulic cylinders to make mold core fluctuation so that can change the gap of mold core. The linear displacement sensor get voltage signal through measuring gap and then feedback the wall thickness programmer. This make up the loop-locked wall thickness control system.

Soft design of blow molding wall thickness control system. The control system on the basis of the products' configuration and wall thickness be needed and get the wall thickness curve through CAD in mapping software then digitalize the curve with $A / D$ transition at last send the signal to control opening of die after operation by the microcomputer system. The software structure diagram as fig.7.

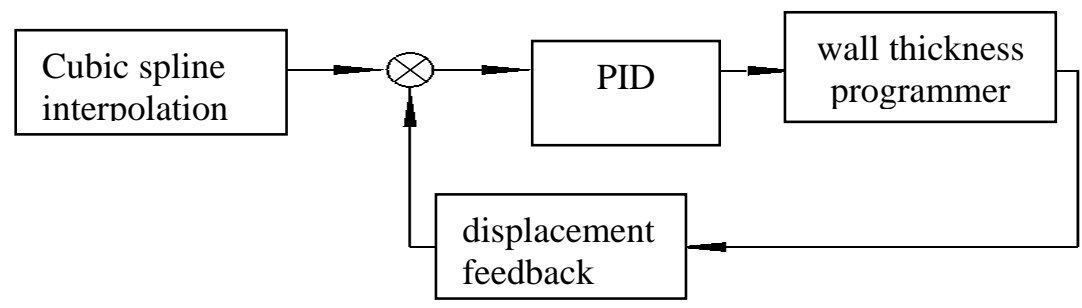

Fig. 7 The software structure diagram

Cubic spline interpolation method used in the touch screen that can create a curve like fig.8.

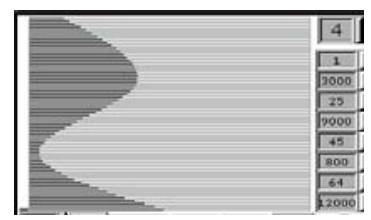

Fig. 8 Wall thickness curve with Cubic spline interpolation method

Hardware selection of blow molding wall thickness control system.In consideration of the reliability, practical applicability and a high cost performance of the system, we choose SIEMENS S7-300 PLC as the control core of the wall thickness control system.S7-300 supply the PID control functional blocks to achieve PID control. We choose SIEMENS MP277 touch screen, 64K color display.

\section{Software design of blow molding machine}

Software design include two parts: program of PLC and design of HMI.

Sequential control program of PLC.As working sequence of demand, PLC estimate the system's condition based on the input signals detected then make each contactors, relays and solenoid valves' open or closed by a series of output signals to control each parts' mechanical action. The 
machine's working process is a sequential control process, when one step of crafts finished check up each sensors and limit switches to decide whether to start the next step.

The control program is divided into 4 styles: automatic single cycle, single step, manual operation and emergency manual operation. All the 4 styles based on the "valves blocks" as its elementary unit. The automatic single cycle operation is make up of different single step operation and the single step operation is make up different manual operation, so the "valves blocks" is the basic programming element. All the working styles establish on it. For example, the under blowing raise up "valves blocks” as fig.9.

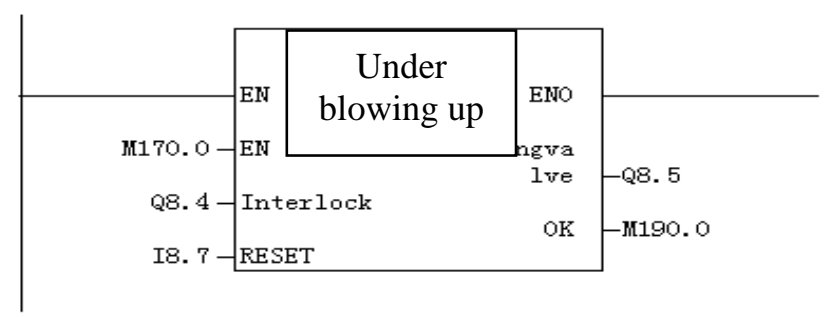

Fig. 9 Under blowing raise up "valves blocks"

In addition, there is a stop switch setting in the control system, there is also setting an alarm function, when a bug appears, HMI shows it and prompt operator to deal with.

Design of human-computer interface.Human-computer interface mainly include starting up picture, main picture and each function pictures. This can make the operators monitoring and modifying the data conveniently.

When the system power on, enter into the main picture, there are many buttons in the main picture to jump into each function pictures, the fig.10,11,12,13 is the main picture and some function pictures.

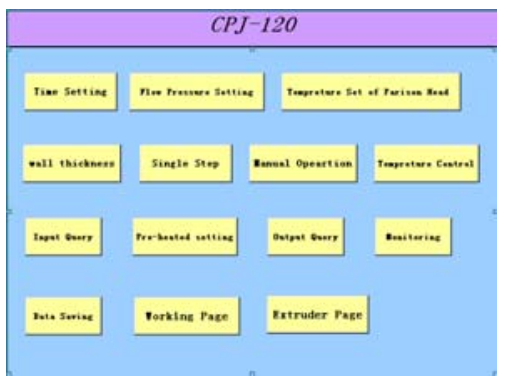

Fig. 10 Main picture

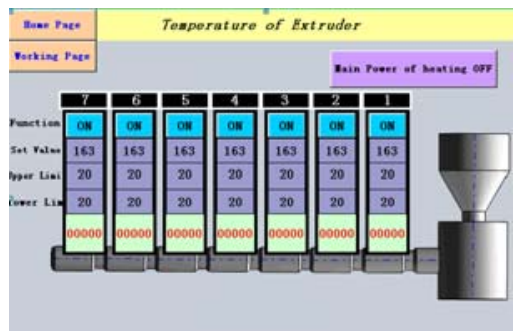

Fig. 12 Temperature of Extruder

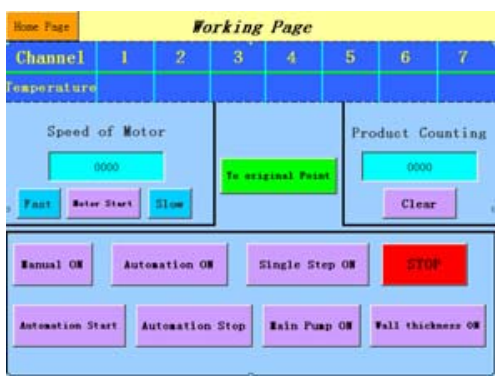

Fig. 11 Working picture

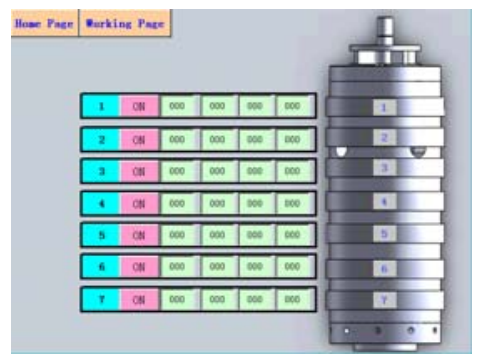

Fig. 13 Temperature of parison head

In addition, there are also some pictures to supervise the system and may reflect errors or bugs when the machine is moving.

\section{Conclusions}

This blow molding sequence control system after debugging and moving, each working condition may achieve the designed goal completely, as adopt sequence control model, debugging is very 
convenient. The parison wall thickness programmer make the thickness of parison reasonable and achieve the technology index that the thickness is uniformity. This may reduce the consumption of raw materials substantially.

\section{Acknowledgments}

The Authors are grateful for support of science and technology found of Liaoning Province, No. 2011216012.

\section{References}

[1] Changchu Liao:Follow Me to Learn S7-300/400 PLC. (China Machine Press,2011.2)

[2] Hanxiong Huang: Plastic Blow Molding Technology (Chemical Industry Press (CIP), Beijing 1995)

[3] YouGen Zhang: Prolongation of Blow Molding Machine. (HaiDa Plastic machinery Co, LTD,Ningbo 2009)

[4] XiaoHan Xiang, Bin Lu:Foundation And Case of S7-300/400 PLC. (China Machine Press,2011)

[5] Changchu Liao: Configuration And Application of Siemens HMI. (China Machine Press,2008)

[6] ZiKangWang:Research And Design of Parison Wall Thickness Programmer.(Chongqing Univerity,2006)

[7] I. Dobson, B. A. Carreras, V. E. Lynch, D. E. Newman,An initial model for complex dynamics in electric power system blackouts,(Hawaii International Conference on System Sciences, Maui, Hawaii,2005.1) 